\title{
Biannually School Based Deworming by Mebendazole 500mg has Reduced the Worm Load Drastically in Bangladesh
}

\author{
Muhammad Mujibur Rahman* \\ Ministry of Health and Family Welfare, Bangladesh \\ Submission: March 03, 2017; Published: May 24, 2017 \\ *Corresponding author: Muhammad Mujibur Rahman, Consultant, Filariasis Elimination and STH Control Program, Ministry of Health and Family \\ Welfare, Bangladesh, Email: drmujib.rahman@gmail.com
}

\section{Perspective}

Globally, national deworming programs utilize a mass drug administration (MDA) approach for the delivery of deworming pills to all school age children. Programs which deliver deworming pills to all school-age children on a regular basis is not only treat the infected individuals, but also help to reduce the community burden. This results in lower worm loads overall and less re-infection. The drugs used to treat the most common STH infections are safe, effective, economical and easy to administer [1]. The World Health Organization (WHO) has set global goals to reach 75 to $100 \%$ of school age children in need (WHO Helminth Control in School Age Children). USAID's Global Health Initiative (GHI) aims to reduce the prevalence of seven neglected tropical diseases (NTD) by 50 percent among 70 percent of the affected population.

Bangladesh being in the tropical zone and having all the risk factors of all parasitic diseases harbors the biggest load of the parasitic disease burden. All 64 districts are endemic with 78 million infected with Ascaris lumbricoides (Round worm) which constituted $55 \%$ of population. About 51 million people (35\% of population) are infected with Hook worm and 66 million people are infected with Trichuris trichiura (Whip worm) [1,2]. Among all the community 5-14 years age group are very vulnerable to the infection. It causes impairment of growth, development of cognition among Children. Hook worm and whip worm infection cause iron deficiency anaemia.

Prior to the initiation of programmatic activities, schools in three districts began piloting deworming programs in 2005 covering three ecological zones of the country (plain, coastal and hilly). The results found $79.8 \%$ of the Children were infected. In addition, $43.6 \%$ had moderate heavy intensities of Ascaris infections and $16.2 \%$ had moderate heavy intensities of Trichuris trichiura infections.
Another follow up survey was conducted in 2007 in same places adding three more districts where deworming was done during MDA under Filariasis elimination program. The three districts were Panchagarh, Nilphamari and Lalmonirhat. The sample size was 1515 . The following results were observed. $76.02 \%$ school children were found with no infection, $18.65 \%$ with single, $4.91 \%$ with double and $0.39 \%$ with triple infection.

On the basis of that survey results, the government of Bangladesh estimated that 20 million children were at risk for STH. In response to this, the Government of Bangladesh's Ministry of Health established deworming programs through the National Filariasis Elimination with the support of various partners including the WHO, Children Without Worms (CWW) and Johnson \& Johnson.

School based deworming program was scaled up gradually to 16 districts till 2006 to June 2007, 24 districts till May 2008 and finally it was expanded up to 64 districts by November 2008 with an aim to regular de-worming of $75-100 \%$ School aged children (WHA resolution 54.19 of 2001). Deworming is now conducted for all school aged5-12 years old, through all primary level institutions (govt. and non-govt.) in the country. The first national de-worming day was observed on 1 November 2008. Subsequently the program was implemented every six months, May \& November.

From 2010 De-worming is conducted for a week instead of National de-worming Day and 5 years aged (baby class/preprimary) group included as target. Deworming week has been shifted from May to April and from November to October during the year 2013. Single dose of tablet Mebendazole (500mg) instead of Tablet Albendazole is being used countrywide in deworming program from the November round of 2012. Children without worms (CWW) a USA based organization have 
been donating Tablet Mebendazole (500mg) for whole country. In November round of 2008 , the total targeted children were 15, 743, 159 and treated 15, 482, 778 with 93.64\% reported coverage. In the October round of 2015 the number of targeted children reached to $2,72,49,461$ of which 2, 70, 83, 502 treated with $99.39 \%$ reported coverage. The current figure of targeted children of Bangladesh STH control program might correspond to the total population of a small country of the world.

Program data shows very high levels of reported coverage, [3] although actual pill ingestion may be less than reported according to the report of the post MDA validation survey organized by CDC Atlanta in 2010 in Munshigonj and Laxmipur districts. According to recommendation of that survey, MDA should be accompanied by IEC, advocacy, and training aimed at educating the public about soil transmitted helminths. Although the program has been successfully rolled out, it has suffered from certain gaps and compliance issues which undermine the quality and hence effectiveness.

To address these gaps FHI 360 with funding from USAID's END in Asia Project began providing support for specific activities at the request of the national program for STH Control in 2011. The overall goal is to strengthen and expand national NTD control efforts. More over a "Little Doctor" initiative was started from the same year with an aim to increase drug coverage as well as providing messages of some important health related matters to other students' especially personal hygiene. Topic on personal hygiene is built in their text books as well as in the flipchart provided to each team of Little Doctors of whole country. It is one of their responsibilities of the Little Doctor team to teach and demonstrate personal hygiene practices in school like hand washing after defecation, wearing sandal or shoes during use of latrine and not to walk on bare footed. Not to eat any fresh fruit without washing with safe water and not to take any meal without washing hands properly. Five tips for washing hands are also being taught and practiced in the school. The Little Doctor initiative was highly accepted by the schools and their role was appreciated by every individual nationally and internationally.

Deworming alone could not control helminthes infestation of a country unless health and hygiene practices are in place. On the basis of that idea, "wash blocks" facilities have been implementing in all type of government and non-government schools of the country with supplying of hand washing detergents. As a result of that the subsequent follow-up stool surveys showed STH infection rate $27.2 \%$ in $2010,15.7 \%$ in 2012-2013 and $8.28 \%$ in $2014-2015$ from the $79.8 \%$ baseline infection rate. These joint efforts directly contribute to global goals to establish control of worm infections [4].

\section{Acknowledgement}

The author extends his heartfelt thanks to the staff members of Filariasis Elimination and STH Control Program, Bangladesh who did a lot of efforts in field to complete their survey. He is also grateful to all of them for their support.

\section{References}

1. Report on Baseline Survey on STH among school children (2005) Filariasis Elimination Program, Directorate General of Health Services, Ministry of Health and Family Welfare, Bangladesh.

2. Ministry of Health and Welfare (2010) A situational Analysis: Neglected Tropical Diseases in Bangladesh, Government of the People's Republic of Bangladesh, pp. 1-66.

3. World Health Organization (2011) Elimination of Lymphatic Filariasis in the South-East Asia Region, Report of the eighth meeting of the regional programme review group (RPRG), Colombo, Sri Lanka, pp. 26.

4. Report on Survey on STH among school children (2014-15) Filariasis Elimination Program, Directorate General of Health Services, Ministry of Health and Family Welfare, Bangladesh.

This work is licensed under Creative Commons Attribution 4.0 Licens DOI: 10.19080/AIBM.2017.03.555617

\section{Your next submission with Juniper Publishers will reach you the below assets}

- Quality Editorial service

- Swift Peer Review

- Reprints availability

- E-prints Service

- Manuscript Podcast for convenient understanding

- Global attainment for your research

- Manuscript accessibility in different formats

( Pdf, E-pub, Full Text, Audio)

- Unceasing customer service

Track the below URL for one-step submission https://juniperpublishers.com/online-submission.php 